\title{
Regular physical education class enhances sociality and physical fitness while reducing psychological problems in children of multicultural families
}

\author{
Jae-Wan Park', Seong-Hwan Park', Chang-Mo Koo 1,2, Denny Eun ${ }^{3}$, Kang-Ho Kim³', Chan-Bok Lee ${ }^{4}$, Joung-Hyun Ham², \\ Jeong-Hoon Jang ${ }^{2}$, Yong-Seok Jee,** \\ 'Department of Education (Major of Physical Education), Graduate School of Education, Hanseo University, Seosan, Korea \\ ${ }^{2}$ Research Institute of Sports and Industry Science, Hanseo University, Seosan, Korea \\ ${ }^{3}$ Department of Public Health . Special Education, Graduate School of Health Promotion, Hanseo University, Seosan, Korea \\ ${ }^{4}$ Department of International CKD Martial Art, Graduate School of Health Promotion, Hanseo University, Seosan, Korea
}

This study investigated the influence of physical education class (PEC) as an intervention method for aggression, sociality, stress, and physical fitness levels in children from multicultural families. The hypothesis was that participating in PEC would result in reduced aggression and stress and improved sociality and physical fitness in multicultural children. A three-item questionnaire, a body composition test, and physical fitness tests were given three times. Eighty-four subjects were divided into four groups: multicultural children who participated in PEC (multi-PEG, $n=12$ ), multicultural children who did not participate in PEC (multiNPEG, $n=13$ ), single-cultural children who participated in PEC (singPEG, $n=11$ ), and single-cultural children who did not participate in PEC (sing-NPEG, $n=12$ ), respectively. Parametric and nonparametric statistical methods were conducted on the collected data with a significance level set a priori at $P<0.05$. After 8 weeks of $P E C$, fat mass $(F=2.966$, $P=0.045)$ and body mass index $(F=3.654, P=0.021)$ had significantly dif-

\section{INTRODUCTION}

We now live in a global society in which people from diverse backgrounds live and interact. This multicultural society contains a mixture of the unique cultures of many nations. Due to the globalization wave in the early 1990s, the number of foreign workers in Korea increased due to frequent exchanges with foreign countries. Around this period, there was an increasing number of single men, especially in rural areas of Korea, who have been unable to find a spouse. As more and more foreign women were wel- ferent interaction effects. In the aspect of interaction effects from physical fitness variables, cardiopulmonary endurance $(F=21.961, P=0.001)$, flexibility $(F=8.892, P=0.001)$, muscular endurance $(F=31.996, P=0.001)$, muscular strength $(F=4.570, P=0.008)$, and power $(F=24.479, P=0.001)$ were significantly improved in the multi-PEG compared to those of the other three groups. Moreover, sociality ( $F=22.144, P=0.001)$ in the multiPEG was enhanced, whereas aggression $(F=6.745, P=0.001)$ and stress $(F=3.242, P=0.033)$ levels were reduced. As conclusion, the PEC reduced aggression and stress levels, and improved sociality and physical fitness levels after 8 weeks. This study confirmed that PEC for children from multicultural families can improve psychosocial factors and physical health.

Keywords: Aggression, Multicultural family, Physical education class, Sociality, Stress
${ }^{*}$ Corresponding author: Yong-Seok Jee (iD http://orcid.org/0000-0001-6797-0843 Research Institute of Sports and Industry Science, Hanseo University, 46 Hanseo 1-ro, Haemi-myeon, Seosan 31962, Korea

Tel: +82-41-660-1028; Fax: +82-41-660-1088. E-mail: jeeys@hanseo.ac.kr

Received: March 3, 2017 / Accepted: March 31, 2017 comed as brides, the number of multicultural families has risen (Kim, 2006).

As children from multicultural families reach school age, they are twice as likely to experience bullying. The fact that their parents were foreigners was the most common reason for being bullied or discriminated against. Since this is an unchangeable circumstance, a serious psychological problem exists for children from multicultural families that can cause them to quit school (Park, 2003). Serious personal problems can result if children are not able to grow up normally, so specialized education for multi- 
cultural families is becoming more important (Kim, 2011).

According to Banks (2013), the objectives of multicultural education are to reduce discrimination experienced by students with diverse cultural backgrounds and to provide all students with equal educational opportunities. Multicultural education is defined as all educational activities that enable students to develop their abilities to actively cope with discrimination and conflicts arising from cultural differences, form amicable relationships, and increase understanding of diverse cultures, ethnic groups, races, genders, and regions (Park, 2000).

Among the various educational activities, physical education class (PEC) plays a big role in inducing cognitive, emotional and psychological changes in children through physical activity. Moreover, although more research is needed on the association between physical activity and health among young people (Armstrong and Simons-Morton, 1994; Bar-Or and Baranowski, 1994), evidence has shown that physical activity results in health benefits for children and adolescents. One of the most preferred subjects is Physical Education because it can encourage children to look past their differences. Children from multicultural families often have difficulty in subjects such as Korean language classes and social studies, but they have least difficulty in physical education. Therefore, PEC can help them adapt to school (Kwon, 2008). However, to date, there have been no specific studies on how PEC and physical fitness can reduce aggression, improve sociality, decrease stress levels in children from multicultural families. Therefore, this study was conducted to investigate the influence of PEC on aggression, sociality, and stress levels and to investigate the effects of physical fitness on those variables in multicultural children.

\section{MATERIALS AND METHODS}

\section{Participants}

The study sample consisted of 50 children ( 25 male, 25 female) who participated in PEC between September 2016 and November of 2016 at C-elementary school located in Seosan city. Among the participants, two subjects, one male and one female, were excluded due to an insufficient amount of time participating in PEC. After exclusion, 48 subjects were included in the final analysis. The subjects were divided into four groups: multicultural children who participated in PEC (multi-PEG, $\mathrm{n}=12$ ), multicultural children who did not participate in PEC (multi-NPEG, $\mathrm{n}=13$ ), single-cultural children who participated in PEC (singPEG, $n=11$ ), and single-cultural children who did not participate in PEC (sing-NPEG, $\mathrm{n}=12$ ). Complete subject characteristics are illustrated in Table 1.

\section{Experimental design}

All procedures were explained to the participants in detail prior to testing. All subjects signed an informed consent form that was approved by the Health Science Human Studies Committee of Hanseo University and Sahmyook University (2-1040781-AB-N01-2016121HR). The subjects later returned to the laboratory to complete the questionnaires (aggression, sociality, and stress), body composition assessments, and physical fitness tests on September 23, 2016 (week 0) before beginning the 8-week PEC. They then repeated this process on October 21, 2016 (end of week 4) and again on November 18, 2016 (end of week 8), respectively.

\section{Measurement methods \\ Aggression scale}

The aggression scale measures the intention or motivation of certain behaviors and thoughts which can be defined as socially aggressive or harmful. The questionnaire used in this study was comprised of 30 questions screened from the original 45 questions through factor analysis: 16 questions on behavioral aggression, eight questions on hostility, and six questions on social anger (Ta-

Table 1. Physical characteristics of the subjects

\begin{tabular}{lcccccc}
\hline Characteristic & Multi-PEG $(\mathrm{n}=12)$ & Multi-NPEG $(\mathrm{n}=13)$ & Sing-PEG $(\mathrm{n}=11)$ & Sing-NPEG $(\mathrm{n}=12)$ & $\chi^{2}$ & $P$-value \\
\hline Age $(\mathrm{yr})$ & $11.60 \pm 0.84$ & $12.10 \pm 0.56$ & $12.50 \pm 0.52$ & $12.50 \pm 0.70$ & 9.073 & 0.028 \\
Height $(\mathrm{cm})$ & $147.60 \pm 9.66$ & $151.40 \pm 6.80$ & $151.00 \pm 4.52$ & $153.90 \pm 9.56$ & 3.477 & 0.324 \\
Weight $(\mathrm{kg})$ & $50.87 \pm 13.28$ & $48.72 \pm 14.72$ & $45.87 \pm 10.46$ & $53.60 \pm 18.00$ & 1.181 & 0.757 \\
Percent fat $(\%)$ & $33.87 \pm 8.05$ & $25.14 \pm 7.18$ & $23.81 \pm 13.06$ & $28.16 \pm 11.78$ & 5.594 & 0.133 \\
\hline
\end{tabular}

Values are presented as mean \pm standard deviation.

Results from Kruskal-Wallis test by nonparametric tests. The multi-PEG, multi-NPEG, sing-PEG, and sing-NPEG represents multicultural children group that participated in PEC, multicultural children group that did not participate in PEC, single-cultural children group that participated in PEC, and single-cultural children group that did not participate in PEC, respectively.

PEG, physical education group; NPEG, nonphysical education group. 
Table 2. Items of aggression, sociality, and stress scales and Cronbach $\alpha$

\begin{tabular}{|c|c|c|}
\hline \multirow{2}{*}{ Factor } & \multirow{2}{*}{ Number } & \multirow{2}{*}{$\begin{array}{c}\text { Cronbach } \alpha \\
\text { Subfactor }\end{array}$} \\
\hline & & \\
\hline Aggression & & 0.91 \\
\hline Behavioral aggression & $1-14,20,24$ & 0.90 \\
\hline Hostility & $26,27,29-32,34,35$ & 0.91 \\
\hline Social anger & $39,41-45$ & 0.83 \\
\hline Sociality & & 0.88 \\
\hline Interrelationship & $1,5,6,13,18,23,27,31,32,36$ & 0.87 \\
\hline Responsibility & $2,7,10,14,19,24,28,33,37,40$ & 0.85 \\
\hline Cooperation & $3,8,11,15,17,20,25,29,34,38$ & 0.83 \\
\hline Diligence & $4,9,12,16,21,22,26,30,35,39$ & 0.87 \\
\hline Stress & & 0.88 \\
\hline Friendships & $1-7$ & 0.83 \\
\hline Credit & 8-14 & 0.87 \\
\hline School and teachers & $15-21$ & 0.88 \\
\hline
\end{tabular}

ble 2) (Lee, 2011). Point scores of 1-5 were used to indicate the following answers: 'never' (1), 'almost never' (2), 'some of the time' (3), 'most of the time' (4), and 'almost always' (5). These values were assigned and aggregated to determine the degree of aggressiveness and harmful behaviors. The reliability of the questionnaire was measured by calculating Cronbach $\alpha$, representing internal consistency. The values of questions including behavioral aggression, hostility, and social anger were distributed in the range from a minimum of 0.83 to a maximum of 0.91 .

\section{Sociality scale}

The sociality scale covers the intention or motivation of certain behaviors. The questionnaire used in this study was comprised of 40 questions screened from the original 40 questions through factor analysis: 10 questions on interrelationships, 10 questions on responsibility, 10 questions on cooperation, and 10 questions on diligence (Table 2) (Min, 2005). Point scores of 1-5 were identical to the aggression scale. These values were assigned and aggregated to determine the degree of social behaviors. The reliabilities of the questionnaire measured by calculating Cronbach $\alpha$ were distributed in the range from a minimum of 0.83 to a maximum of 0.87 .

\section{Stress scale}

The stress scale measures the intention or motivation of certain thoughts and actions which can be defined as stressful factors. The questionnaire used in this study was comprised of 21 questions screened from the original 21 questions through factor analysis: seven questions on relationships, seven questions on credit factors, and seven questions on school and teachers (Kang, 2004). Point scores of $1-5$ were also identical to the aggression scale. These values were assigned to determine the degree of stress. The reliabilities of the questionnaire assessed by calculating Cronbach $\alpha$ were distributed in the range from a minimum of 0.83 to a maximum of 0.88 (Table 2).

\section{Physical fitness test}

The specific measurement methods of physical fitness tests were as follows.

\section{Measurement of body composition}

Body composition measurements including height, weight, skeletal muscle mass, body fat mass, body fat percentage, body mass index, and basal metabolic rate were performed by bioelectrical impedance analysis using InBody 230 (Biospace, Seoul, Korea), which is regarded as a simple, inexpensive, and reliable method for adult men and women (Janssen et al., 2002). BMS 330 anthropometer (BioSpace) was used to measure the height of subjects. The Body Composition Analyzer is a segmental impedance device measuring the voltage drop in the upper and lower body. The electrodes are made of stainless steel and the electrical interfaces were created as the subject stood upright while gripping hand electrodes and stepping onto foot electrodes. Eight tactile electrodes were placed in contact with the surfaces of both hands and feet: thumb, palm and fingers, front sole, and rear sole (Pil-Byung et al., 2011). Body weight, muscle mass, and body fat mass were expressed in kilograms $(\mathrm{kg})$. Fat percentage was expressed in \%. Analysis of body composition was measured before dinner and after voiding (Cha et al., 2014; Kim et al., 2015).

\section{Measurement of cardiopulmonary endurance}

To measure the 'aerobic capacity' of the subjects, a run/walk test for a specified distance $(1,000 \mathrm{~m})$ was used. Their aerobic capacity was assessed based on the time of completion. A distance of 1,000 $m$ on a school track was measured and a stopwatch was used to time the test. The greatest value after one measurement was recorded.

\section{Measurement of flexibility}

Taking into consideration the children's safety, a sit and reach flexion test was used to measure flexibility. It was performed as follows: A test platform with a ruler $(25 \mathrm{~cm}$ upwards, $40 \mathrm{~cm}$ downwards) attached perpendicularly to the surface was prepared. The subject sat with their heels against the platform with both feet about $5 \mathrm{~cm}$ apart. The subjects took a deep breath and bent forward while exhaling so that the fingertips reached the furthest 
point possible. The subjects were instructed not to bounce the waist forward while bending. The subjects kept their heads between both arms when bending forward. The subjects did not bend their knees. The greatest value after two measurements was recorded.

\section{Measurement of muscle endurance}

A sit-up test was used to measure muscle endurance. The specific methods were as follows: Subjects lied down on a mat with the feet about $30 \mathrm{~cm}$ apart. An assistant held both ankles down. The knees were bent at $90^{\circ}$ with both hands locked behind the head. At the starting signal, the upper body bent forward so that the elbows touched the knees. One sit-up was counted if both elbows touched the knees and the back touched the floor. The greatest number of sit-ups during 1 min was recorded.

\section{Measurement of muscle strength}

Grip strength test was used to measure muscle power. Grip strength, which is the maximum strength of the combined coordination of the thumb and four fingers, was measured using a Smedley dynamometer. Muscle units related with grip strength included the flexors of the anterior brachial muscles and the hand muscles. Forearm strength was measured as follows: The dynamometer was held so that the proximal interphalangeal joint was at a right angle with the width adjusted accordingly. The arms were placed on the sides in a natural resting position with the dynamometer not touching the body. Both hands were consecutively tested twice with the maximum value being recorded. The following instructions were given to the subjects for squeezing the handlebar as hard as possible. The arm may be bent or straightened, but the hand, elbow, or arm should not touch the body. Perform with both right and left hands.

\section{Measurement of power}

A 50-m dash was used to measure power. It is a relatively uncommon nonchampionship event for indoor track and field in which participants sprint for the entire distance of $50 \mathrm{~m}$. The anaerobic capacity of the subjects was assessed based on the time of completion. A distance of $50 \mathrm{~m}$ on a school track was measured and a stopwatch was used to time the test. The greatest value after one measurement was recorded.

\section{Programs of PEC}

Subjects in multi-PEG and sing-PEG have taken part in supervised progressive PEC program for 8 weeks (Table 3). During this
Table 3. Programs of physical education class for exercise groups

\begin{tabular}{|c|c|c|c|}
\hline Type & Periods & Program orders & $\begin{array}{l}\text { Exercise reps (or time) } \\
\quad \times \text { rest time } \times \text { set }\end{array}$ \\
\hline Warm-up & Weeks 1-8 & Stretching of 8 types & $10-15 \min \times 10 \mathrm{sec} \times 1 \mathrm{set}$ \\
\hline \multirow{6}{*}{$\begin{array}{l}\text { 1st work-out } \\
\text { phase }\end{array}$} & \multirow{6}{*}{ Weeks 1-2 } & Squat & 10 reps $\times 10 \sec \times 3$ sets \\
\hline & & Burpee & 10 reps $\times 10$ sec $\times 3$ sets \\
\hline & & Step-box & $5 \mathrm{~min} \times 0 \mathrm{sec} \times 1$ set \\
\hline & & Lunge & 10 reps $\times 1 \mathrm{~min} \times 3$ sets \\
\hline & & Plank & $1 \mathrm{~min} \times 10 \mathrm{sec} \times 3$ sets \\
\hline & & Shuttle run & $10 \mathrm{~min} \times 0 \mathrm{sec} \times 1 \mathrm{set}$ \\
\hline \multirow{6}{*}{$\begin{array}{l}\text { 2nd work-out } \\
\text { phase }\end{array}$} & \multirow[t]{6}{*}{ Weeks 3-5 } & Squat & 12 reps $\times 10$ sec $\times 3$ sets \\
\hline & & Burpee & 12 reps $\times 10$ sec $\times 3$ sets \\
\hline & & Step-box & $5 \mathrm{~min} \times 0 \mathrm{sec} \times 1$ set \\
\hline & & Lunge & 12 reps $\times 1 \mathrm{~min} \times 3$ sets \\
\hline & & Plank & $2 \mathrm{~min} \times 10 \sec \times 3$ sets \\
\hline & & Shuttle run & $15 \mathrm{~min} \times 0 \mathrm{sec} \times 1$ set \\
\hline \multirow{6}{*}{$\begin{array}{l}\text { 3rd work-out } \\
\text { phase }\end{array}$} & \multirow[t]{6}{*}{ Weeks 6-8 } & Squat & 15 reps $\times 10 \sec \times 3$ sets \\
\hline & & Burpee & 15 reps $\times 10 \sec \times 3$ sets \\
\hline & & Step-box & $5 \min \times 0 \sec \times 1$ set \\
\hline & & Lunge & 15 reps $\times 1 \mathrm{~min} \times 3$ sets \\
\hline & & Plank & $3 \mathrm{~min} \times 10 \mathrm{sec} \times 3$ sets \\
\hline & & Shuttle run & $20 \min \times 0 \sec \times 1$ set \\
\hline Cool-down & Weeks 1-8 & Stretching of 8 types & $10-15 \mathrm{~min} \times 10 \mathrm{sec} \times 1 \mathrm{set}$ \\
\hline
\end{tabular}

experimental period, all subjects agreed not to change their daily activity patterns outside of their participation in this study. Subjects also agreed not to change their dietary habits throughout the study period. Subjects performed a warm-up and cool-down program including 8 types of stretching motions for 10-15 min before and after the exercise session.

This was followed by the 1st workout phase (week 1-week 2), which involved squats, burpees, step-box exercises, lunges, planks, and shuttle run exercises at an intensity of less than 13 (somewhat hard) on the ratings of perceived exertion (RPE) scale. The goal of this stage focused on adjusting to the new physical activity and movements involving body weight. The subjects then performed the 2nd workout phase (week 3-week 5) in which the exercises involved in the 1st workout phase was increased in intensity (RPE, 13-15) and repetition. The goal of this stage focused on tolerating full body-weight training and improving cardiopulmonary capacity. The subjects performed the 3 rd workout phase (week 6-week 8) in which the exercises involved in the 2nd workout phase was increased in intensity (RPE, 15 over) and repetition. The goal of this stage focused on increasing cardiopulmonary capacity and maintaining their enhanced physical fitness levels.

Squats used in the workout were a compound, full body exercise that primarily trained the muscles of the thighs, hips and 
buttocks, quadriceps, and hamstrings, as well as strengthening the bones, ligaments and tendons throughout the lower body. Squats were considered a vital exercise for increasing the strength and size of the legs and buttocks in addition to developing core strength. Burpees are a type of aerobic exercise that can maximize exercise effects in a short amount of time. It has recently attracted much attention and is often used as a fitness test. It is a high intensity exercise that can improve physical strength. Moreover, step-box exercise has been scientifically proven to be effective and has become a popular form of aerobics training. It is based on aerobic conditioning and includes weight training and rhythmic movements to develop muscular strength. Step-box exercises are easy to perform and effective for exercising the calf and thigh muscles as an alternative to running. It also plays a role in heart function by including upper body exercises. These step-box exercises use balanced movements that result in the same exercise effects on each part of the body. It is a safe, fun, and effective exercise for both men and women that can induce the continuous and rhythmic movements of the muscles. It can also be performed in a small space in any location. It is also a low impact and high intensity exercise compared to other aerobic exercises such as walking, running, and swimming. In addition, it includes rhythmic movements that strengthens and develops cardiopulmonary endurance and muscle strength. According to the American College of Sports Medicine (2000), the recommended duration for aerobic exercise typically ranges from 15 to $60 \mathrm{~min}$. The ACSM also recommends exercising 3 to 7 times per week and reaching an exercise intensity of $60 \%$ to $90 \%$ of one's maximum heart rate. Performing lunges strengthens the thighs, hips, and lower body. Lunges can be performed solely using body weight. After lunge exercises, the instructor taught plank exercises to the subjects. Lastly, the instructor had the subjects perform a shuttle run, which is a type of cardiopulmonary endurance exercise with increasing load (Léger and Lambert, 1982). Not only does it provide a reasonable estimate of the maximal oxygen uptake, it is not as tedious as long-distance running. It is possible to minimize the occurrence of safety accidents because it is adjustable according to ability. The objective of a shuttle run was to increase the maximum oxygen uptake to increase endurance and power (Léger and Gadoury, 1989). Meanwhile, the 50-m dash was used to improve the anaerobic capacity (the maximal accumulated oxygen deficit), which can be developed through high-intensity intermittent training (Tabata et al., 1996).

\section{Statistical analyses}

All data are reported as mean \pm standard deviation and as mean change over baseline (95\% confidence interval). The KolmogorovSmirnov test was used to determine the normality of distribution of the examined variables. Differences in baseline characteristics among the four groups of children were evaluated with two-way $(4 \times 3)$ repeated measured analysis of variance and with the Kruskal-Wallis test for normally and nonnormally distributed variables, respectively. Repeated measures and analysis of variance controlling for age was used to evaluate the significance of the differences among groups at baseline (week 0), at midtest (week 4) and at the end (week 8) of the 8-week intervention (treatment effect) program; the significance of the changes observed within each group (time effect); and the effect of treatment $\times$ time interaction. The between-group factor was the study groups (i.e., multi-PEG vs. multi-NPEG vs. sing-PEG vs. sing-NPEG); the within-group factor was the time-point of measurement. Specifically, the MannWhitney test was used to determine the difference of variables between multi-PEG and multi-NPEG or between sing-PEG and sing-NPEG. All $P$-values reported were two-tailed. Statistical analysis was conducted with the SPSS ver. 18.0 (SPSS Inc., Chicago, IL, USA). The level of statistical significance was set at $P<0.05$.

\section{RESULTS}

\section{Influence of PEC on body composition in children}

Table 4 shows the body composition among the four groups (multi-PEG, multi-NPEG, sing-PEG, and sing-NPEG) after 8 weeks of PEC. The data from those groups were analyzed for differences and changes after taking part in PEC. Table 4 displays the differences and changes observed in the body composition levels of the four groups. As shown in the table, only fat mass $(F=2.966, P=0.045)$ and BMI $(F=3.654, P=0.021)$ were significantly different in interaction effects. In other words, the 8 weeks of PEC may be associated with the loss of fat in the multiPEG than those of the other three groups.

\section{Influence of PEC on physical fitness levels in children}

Table 5 shows the physical fitness levels among the four groups (multi-PEG, multi-NPEG, sing-PEG, and sing-NPEG) after 8 weeks of PEC. The data from those groups were analyzed for differences and changes after taking part in PEC. Table 5 displays the differences and changes observed in the physical fitness levels of the four groups. As shown in the table, cardiopulmonary endurance was significantly different between groups $(F=3.689$, 
Park JW, et al. • Physical activity improves physical fitness and reduces psychological problems

Table 4. Differences and changes of body composition among four groups after PEC

\begin{tabular}{|c|c|c|c|c|c|c|c|c|}
\hline \multirow{2}{*}{ Factor } & \multirow{2}{*}{ Week } & \multirow{2}{*}{ Multi-PEG } & \multirow{2}{*}{ Multi-NPEG } & \multirow{2}{*}{ Sing-PEG } & \multirow{2}{*}{ Sing-NPEG } & \multicolumn{3}{|c|}{$F(P \text {-value })^{*}$} \\
\hline & & & & & & Group (G) & Time (T) & $\mathrm{G}^{*} \mathrm{~T}$ \\
\hline \multirow[t]{3}{*}{ Weight (kg) } & 0 & $50.87 \pm 13.28$ & $48.72 \pm 14.72$ & $45.87 \pm 10.46$ & $53.60 \pm 18.00$ & $0.543(0.656)$ & $50.814(0.001)$ & $1.344(0.275$ \\
\hline & 4 & $51.07 \pm 12.28$ & $49.86 \pm 14.90$ & $46.43 \pm 10.33$ & $54.53 \pm 18.14$ & & & \\
\hline & 8 & $51.71 \pm 12.88$ & $50.56 \pm 14.59$ & $47.11 \pm 10.25$ & $55.31 \pm 18.18$ & & & \\
\hline \multirow[t]{3}{*}{ Muscle mass (kg) } & 0 & $17.61 \pm 4.61$ & $19.19 \pm 4.70$ & $18.10 \pm 2.71$ & $18.97 \pm 5.42$ & $0.315(0.815)$ & $1.863(0.181)$ & $1.434(0.249$ \\
\hline & 4 & $17.35 \pm 4.55$ & $19.33 \pm 4.93$ & $17.56 \pm 3.16$ & $19.41 \pm 5.19$ & & & \\
\hline & 8 & $18.42 \pm 5.10$ & $18.94 \pm 5.83$ & $18.05 \pm 3.34$ & $19.67 \pm 5.11$ & & & \\
\hline \multirow[t]{3}{*}{ Fat mass (kg) } & 0 & $17.72 \pm 6.99$ & $13.01 \pm 7.47$ & $11.94 \pm 8.06$ & $18.10 \pm 9.78$ & $1.124(0.352)$ & $7.458(0.010)$ & $2.966(0.045$ \\
\hline & 4 & $18.28 \pm 6.28$ & $14.02 \pm 7.59$ & $13.36 \pm 7.31$ & $18.36 \pm 10.94$ & & & \\
\hline & 8 & $17.28 \pm 5.84$ & $15.64 \pm 7.08$ & $13.27 \pm 7.13$ & $18.68 \pm 10.80$ & & & \\
\hline \multirow[t]{3}{*}{$\mathrm{BMI}\left(\mathrm{kg} / \mathrm{m}^{2}\right)$} & 0 & $23.08 \pm 3.85$ & $20.96 \pm 4.66$ & $20.03 \pm 3.99$ & $22.14 \pm 5.36$ & $0.830(0.486)$ & $6.695(0.014)$ & $3.654(0.021)$ \\
\hline & 4 & $23.24 \pm 3.70$ & $21.47 \pm 4.66$ & $20.26 \pm 3.94$ & $22.51 \pm 5.45$ & & & \\
\hline & 8 & $22.86 \pm 3.57$ & $21.53 \pm 4.19$ & $20.20 \pm 3.77$ & $22.55 \pm 5.28$ & & & \\
\hline
\end{tabular}

Values are presented as mean \pm standard deviation.

Results from two-way $(4 \times 3)$ repeated analysis of variance (ANOVA) test. The multi-PEG, multi-NPEG, sing-PEG, and sing-NPEG represents multicultural children who participated in PEC, multicultural children with did not participate in PEC, single-cultural children who participated in PEC, and single-cultural children who did not participate PEC, respectively.

PEC, physical education class; BMI, body mass index.

*Two-way repeated ANOVA.

Table 5. Differences and changes in physical fitness among four groups after PEC

\begin{tabular}{|c|c|c|c|c|c|c|c|c|}
\hline \multirow{2}{*}{ Factor } & \multirow{2}{*}{ Week } & \multirow{2}{*}{ Multi-PEG } & \multirow{2}{*}{ Multi-NPEG } & \multirow{2}{*}{ Sing-PEG } & \multirow{2}{*}{ Sing-NPEG } & \multicolumn{3}{|c|}{$F(P \text {-value })^{*}$} \\
\hline & & & & & & Group (G) & Time (T) & $\mathrm{G}^{*} \mathrm{~T}$ \\
\hline \multirow[t]{3}{*}{ Cardiopulmonary endurance (min) } & 0 & $9.62 \pm 2.67$ & $8.25 \pm 2.02$ & $6.68 \pm 1.23$ & $8.83 \pm 2.23$ & $3.689(0.021)$ & $4.544(0.040)$ & $21.961(0.001)$ \\
\hline & 4 & $9.33 \pm 2.56$ & $8.52 \pm 2.27$ & $6.57 \pm 1.19$ & $9.24 \pm 2.38$ & & & \\
\hline & 8 & $9.06 \pm 2.31$ & $8.97 \pm 2.69$ & $6.33 \pm 1.25$ & $9.69 \pm 2.42$ & & & \\
\hline \multirow[t]{3}{*}{ Flexibility (cm) } & 0 & $7.65 \pm 2.63$ & $10.50 \pm 6.34$ & $12.92 \pm 5.45$ & $13.38 \pm 6.90$ & $2.167(0.109)$ & $5.474(0.025)$ & $8.892(0.001)$ \\
\hline & 4 & $7.83 \pm 2.41$ & $10.44 \pm 6.28$ & $13.54 \pm 5.27$ & $13.08 \pm 6.99$ & & & \\
\hline & 8 & $8.78 \pm 2.16$ & $10.26 \pm 6.31$ & $14.38 \pm 5.27$ & $12.67 \pm 6.73$ & & & \\
\hline \multirow[t]{3}{*}{ Muscular endurance (rep/min) } & 0 & $19.70 \pm 11.70$ & $26.30 \pm 11.93$ & $25.50 \pm 12.18$ & $15.40 \pm 13.37$ & $2.097(0.118)$ & $30.945(0.001)$ & $31.996(0.001)$ \\
\hline & 4 & $21.10 \pm 11.70$ & $25.50 \pm 11.87$ & $26.80 \pm 12.11$ & $15.10 \pm 12.95$ & & & \\
\hline & 8 & $23.50 \pm 10.88$ & $24.80 \pm 12.23$ & $30.20 \pm 11.38$ & $14.60 \pm 12.21$ & & & \\
\hline \multirow[t]{3}{*}{ Muscular strength (kg) } & 0 & $17.11 \pm 3.76$ & $20.18 \pm 5.78$ & $18.11 \pm 3.83$ & $20.10 \pm 5.55$ & $0.551(0.650)$ & $2.145(0.152)$ & 4.570 (0.008) \\
\hline & 4 & $17.68 \pm 3.95$ & $20.24 \pm 5.80$ & $18.50 \pm 3.81$ & $20.38 \pm 5.63$ & & & \\
\hline & 8 & $18.32 \pm 4.29$ & $20.27 \pm 5.72$ & $19.46 \pm 4.11$ & $19.00 \pm 5.98$ & & & \\
\hline \multirow[t]{3}{*}{ Power (sec) } & 0 & $10.09 \pm 1.33$ & $9.84 \pm 1.01$ & $9.77 \pm 0.85$ & $10.88 \pm 1.51$ & $3.524(0.024)$ & $1.819(0.186)$ & $24.47(0.001)$ \\
\hline & 4 & $9.95 \pm 1.32$ & $10.07 \pm 1.14$ & $9.59 \pm 0.76$ & $11.13 \pm 1.63$ & & & \\
\hline & 8 & $9.46 \pm 1.01$ & $10.74 \pm 1.61$ & $9.23 \pm 0.86$ & $11.59 \pm 1.53$ & & & \\
\hline
\end{tabular}

Values are presented as mean \pm standard deviation.

Results from two-way $(4 \times 3)$ repeated analysis of variance (ANOVA) test. The multi-PEG, multi-NPEG, sing-PEG, and sing-NPEG represents multicultural children who participated in PEC, multicultural children with did not participate in PEC, single-cultural children who participated in PEC, and single-cultural children who did not participate PEC, respectively.

PEC, physical education class; BMI, body mass index.

${ }^{*}$ Two-way repeated ANOVA.

$P=0.021)$ and between times $(F=4.544, P=0.040)$, respectively. Moreover, significant group $\times$ time interaction effects of cardiopulmonary endurance was observed $(F=21.961, P=0.001)$. Specifi- cally, the flexibility, muscular endurance, muscular strength, and power (Fig. 1) were significantly improved in the multi-PEG than those of the other three groups. 


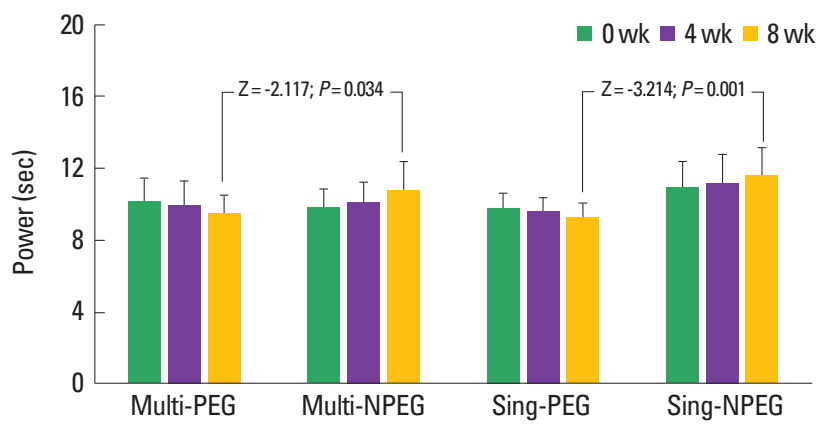

Fig. 1. Differences of power between multi-PEG and multi-NPEG, and between sing-PEG and sing-NPEG after 8 weeks of PEC. These results from the MannWhitney test. The multi-PEG, multi-NPEG, sing-PEG and sing-NPEG represents multicultural children who participated in PEC, multicultural children with did not participate in PEC, single-cultural children who participated in PEC, and single-cultural children who did not participate PEC, respectively.

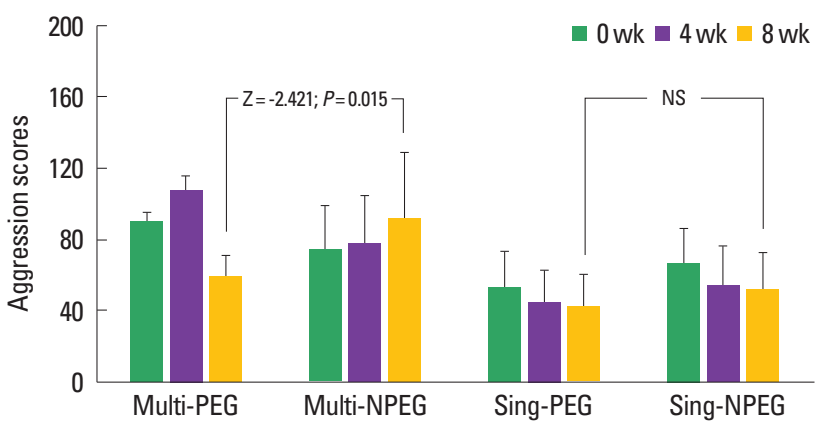

Fig. 2. Differences in aggression levels between multi-PEG and multi-NPEG, and between sing-PEG and sing-NPEG after 8 weeks of PEC. These results from the Mann-Whitney test. The multi-PEG, multi-NPEG, sing-PEG and singNPEG represents multicultural children who participated in PEC, multicultural children with did not participate in PEC, single-cultural children who participated in PEC, and single-cultural children who did not participate PEC, respectively.

Table 6. Differences and changes in aggression, sociality, and stress levels among four groups after PEC

\begin{tabular}{|c|c|c|c|c|c|c|c|c|}
\hline \multirow{2}{*}{ Factor } & \multirow{2}{*}{ Week } & \multirow{2}{*}{ Multi-PEG } & \multirow{2}{*}{ Multi-NPEG } & \multirow{2}{*}{ Sing-PEG } & \multirow{2}{*}{ Sing-NPEG } & \multicolumn{3}{|c|}{$F(P \text {-value })^{*}$} \\
\hline & & & & & & Group (G) & Time (T) & $\mathrm{G}^{*} \mathrm{~T}$ \\
\hline \multirow[t]{3}{*}{ Aggression (score) } & 0 & $90.10 \pm 4.84$ & $73.70 \pm 25.08$ & $52.30 \pm 21.18$ & $66.10 \pm 20.06$ & $12.360(0.001)$ & $6.024(0.019)$ & $6.745(0.001)$ \\
\hline & 4 & $107.00 \pm 8.41$ & $77.50 \pm 27.42$ & $44.80 \pm 17.95$ & $54.20 \pm 21.82$ & & & \\
\hline & 8 & $59.30 \pm 11.63$ & $91.50 \pm 37.51$ & $41.60 \pm 18.63$ & $51.60 \pm 21.11$ & & & \\
\hline \multirow[t]{3}{*}{ Sociality (score) } & 0 & $115.40 \pm 8.80$ & $132.20 \pm 24.84$ & $156.80 \pm 25.34$ & $142.30 \pm 23.74$ & 8.651 (0.001) & $5.988(0.019)$ & $22.144(0.001)$ \\
\hline & 4 & $97.10 \pm 13.05$ & $132.10 \pm 32.45$ & $161.20 \pm 29.88$ & $157.40 \pm 31.20$ & & & \\
\hline & 8 & $154.60 \pm 12.58$ & $95.10 \pm 39.78$ & $174.00 \pm 24.28$ & $156.60 \pm 31.85$ & & & \\
\hline \multirow[t]{3}{*}{ Stress (score) } & 0 & $55.40 \pm 4.93$ & $50.30 \pm 14.81$ & $30.10 \pm 8.03$ & $41.90 \pm 18.43$ & $15.833(0.001)$ & $1.277(0.266)$ & $3.242(0.033)$ \\
\hline & 4 & $67.20 \pm 6.37$ & $50.80 \pm 15.34$ & $27.60 \pm 7.11$ & $38.30 \pm 18.55$ & & & \\
\hline & 8 & $43.40 \pm 6.17$ & $59.80 \pm 21.76$ & $28.80 \pm 12.17$ & $33.90 \pm 15.38$ & & & \\
\hline
\end{tabular}

Values are presented as mean \pm standard deviation.

Results from two-way $(4 \times 3)$ repeated analysis of variance (ANOVA) test. The multi-PEG, multi-NPEG, sing-PEG, and sing-NPEG represents multicultural children who participated in PEC, multicultural children with did not participate in PEC, single-cultural children who participated in PEC, and single-cultural children who did not participate PEC, respectively.

PEC, physical education class; BMI, body mass index.

*Two-way repeated ANOVA.

\section{Influence of PEC on aggression, sociality, and stress levels in children}

Questionnaires were used to analyze aggression, sociality, and stress levels among the four groups (multi-PEG, multi-NPEG, sing-PEG, and sing-NPEG). The data from those groups was analyzed for differences and changes after taking part in PEC (Table 6).

Table 6 displays the differences and changes observed in the aggression levels of the four groups. As shown in the table, significant differences were observed between groups $(F=12.360, P=$ $0.001)$ and between times $(F=6.024, P=0.019)$, respectively. Moreover, significant group $\times$ time interaction effects were observed for aggression scores $(F=6.745, P=0.001)$. These results mean that taking part in PEC decreased aggression levels in both exercise groups (i.e., multi-PEG and sing-PEG). Specifically, the aggression score in the multi-PEG was reduced after 8 weeks, whereas, this score in the multi-NPEG increased. In other words, it was confirmed that participating in PEC decreased the aggression level of children from multicultural families (Fig. 2).

The differences and changes observed in the sociality levels of the four groups are also displayed in Table 6. Significant differences were observed between groups $(F=8.651, P=0.001)$ and between times $(F=5.988, P=0.019)$, respectively. Significant group $\times$ time interaction effects were also observed for sociality scores $(F=22.144$, $P=0.001)$. These results mean that taking part in PEC increased the sociality in both exercise groups (i.e., multi-PEG and singPEG). Specifically, the sociality score in the multi-PEG increased, 


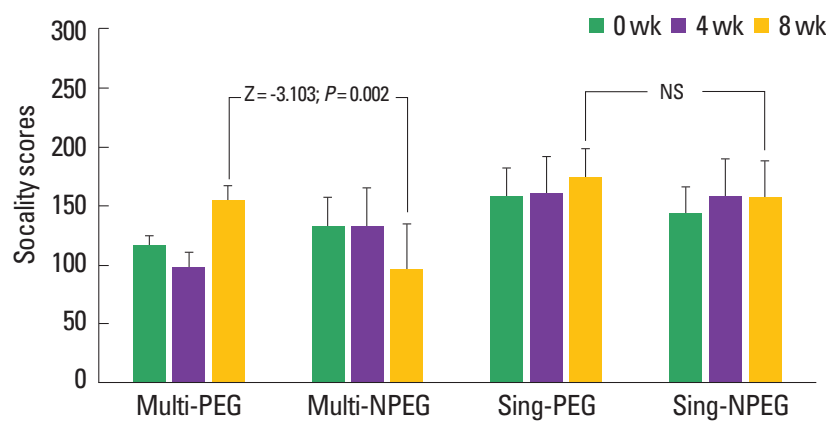

Fig. 3. Differences in sociality levels between multi-PEG and multi-NPEG, and between sing-PEG and sing-NPEG after 8 weeks of PEC. These results from the Mann-Whitney test. The multi-PEG, multi-NPEG, sing-PEG and sing-NPEG represents multicultural children who participated in $\mathrm{PEC}$, multicultural children with did not participate in PEC, single-cultural children who participated in PEC, and single-cultural children who did not participate PEC, respectively.

whereas, this score in the multi-NPEG decreased after 8 weeks as shown in Fig. 3. In other words, it was confirmed that PEC can improve the sociality level of children from multicultural families.

As shown in Table 6, significant differences were observed between groups $(F=15.833, P=0.001)$, except for time $(F=1.277$, $P=0.266)$. However, significant group $\times$ time interaction effects were observed for stress scores $(F=3.242, P=0.033)$. Specifically, the multi-PEG showed an overall decrease in stress scores while the multi-NPEG showed an increase as shown in Fig. 4. However, the decrease observed in stress scores was also found in the sing-PEG and sing-NPEG.

\section{DISCUSSION}

The effects of globalization have significantly increased the number of people from various countries and cultures immigrating to other countries (Berry, 2001; Steffen et al., 2006). Researchers have noted that immigrants have tried to adapt to their new country and culture (Kim et al., 2015; Mori, 2000). However, acculturation stress occurs when the experience of adopting the new culture is perceived as negative. Alienation, anxiety, depression, and sleep disorders are signs and symptoms of acculturation stress (Dixon and Rosenbaum, 2004). These symptoms can be worse in children from multicultural families who are more likely to exhibit higher physical aggression due to communication difficulties. In addition, the troubles that they face when they are in school are serious educational issues. In fact, children of multicultural families in rural areas tend to have lower language abilities than their peers.

If there are several differences between one's own culture and the new culture, adaptation is likely to be more stressful and em-

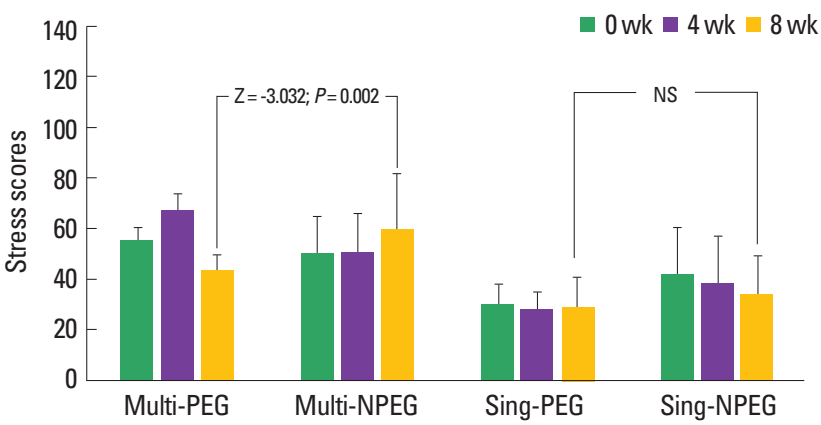

Fig. 4. Differences in stress levels between multi-PEG and multi-NPEG, and between sing-PEG and sing-NPEG after 8 weeks of PEC. These results from the Mann-Whitney test. The multi-PEG, multi-NPEG, sing-PEG and sing-NPEG represents multicultural children who participated in PEC, multicultural children with did not participate in PEC, single-cultural children who participated in PEC, and single-cultural children who did not participate PEC, respectively.

barrassing (Lee et al., 2004; Mclaren, 2003; Tadmor and Tetlock, 2006). When this is experienced daily, it can lead to a significant amount of acculturation stress (Berry, 2005). How children from multicultural families cope with social stigmas is a fertile field for study. Several factors have been identified that deter the detrimental effects of acculturation stress experienced by those immigrating to a foreign country and culture (Rudmin, 2009).

Most researchers agree that physical education programs play important roles in inducing children to make cognitive, emotional, and psychological changes through physical activity. According to a previous study, all adolescents should be physically active daily, or nearly every day, and that adolescents should engage in three or more sessions per week of activities that last $20 \mathrm{~min}$ or more that require moderate to vigorous levels of exertion (Sallis and Patrick, 1994). Recently, researchers have reported that physical activity programs reduce negative psychological symptoms such as loneliness, anxiety, and depression (Bailey and Fernando, 2012; Newman et al., 2013; Wang and Wong, 2014). Other research studies suggest that individuals gain psychological benefits such as personal growth, life satisfaction, happiness, quality of life, and psychological well-being and that physical activity programs, including leisure activities, offer many opportunities for children to enhance positive social interactions with others, which result in psychological benefits (Becchetti et al., 2012; Wang and Wong, 2014). The World Health Organization and the ACSM have also reported that children and adolescents with or without intellectual disabilities can improve their health and quality of life by including a moderate amount of physical activity most days of the week and that additional benefits can be attained with greater amounts of activity (Donnelly et al., 2009; Spear et al., 2007). 
According to the changes observed in aggression and stress levels, which were related to psychological problems in the present study, aggression and stress levels of multi-PEG increased by the 4 th week and decreased on the 8th week. Meanwhile, the aggression and stress levels of multi-NPEG continued to increase from beginning to end. These tendencies showed in the interaction effects of aggression and stress scores, respectively. In the sing-PEG, the aggression scores showed a downward tendency, whereas the stress score showed an increasing tendency. However, these results were not significantly different compared to those of sing-NPEG. In this study, the increases in sociality scores were found to be significantly higher in multi-PEG than the decreases in multiNPEG. However, the sociality scores of sing-PEG were not significantly different compared to those of sing-NPEG. These results suggest that the PEC used in this study can decrease aggression and stress levels and increase sociality in multicultural children.

High levels of stress can lead to a myriad of physical and psychological health issues, but exercise can override the negative effects of stress. Without the need for any prescription medication, simple voluntary physical activity can maintain neurotrophin levels in the brain (Adlard and Cotman, 2004). Physical activity and enhanced growth factor development has been shown to reduce depression and anxiety, as well as strengthening psychological capability for coping with stress (Russo-Neustadt et al., 1999). Such studies strongly suggest the significant influence that exercise can have in mitigating stress and improving behavior which supports the results of this study. Previous studies have also reported that physical activity maintains the structure and function of the body organs, reduces anxiety and depression, enhances social inclusion, and provides a sense of belonging (Dunn et al., 2001). In addition, physical activity can enhance physical, mental and social well-being, as well as the quality of life (Temple and Stanish, 2008). Similar to the results of a previous study, this present study showed a reduction in aggression and stress and improved sociality in children from multicultural families from the participation of physical activity.

Above all, it was thought that the positive psychological changes in multicultural children who participated in PEC were related to improved physical fitness levels. This present study showed that fat mass and BMI significantly improved only in the multiPEG compared to those of the other three groups. According to the changes observed in fat mass and BMI, the multi-PEG increased by the 4th week, but decreased on by the 8th week. These tendencies significantly represented the interaction effects of fat mass and BMI, respectively. In the other three groups, fat mass and BMI showed increased tendencies from the baseline to the fi- nal test. In addition to body composition, the variables of physical fitness in both groups that participated in PEC improved after 8 weeks. Specifically, flexibility, muscular endurance, muscular strength, and power significantly improved in the multi-PEG than those of the other three groups. In other words, the physical education program composed of aerobic and resistance training used in this study positively affected sociality in addition to reducing aggression and stress in multicultural children.

Evidence has shown that regular physical activity improves aerobic endurance (Dotson and Ross, 1985; Pate and Ross, 1987), muscular strength (Sallis et al., 1993), and body mass index (Berkowitz et al., 1985; Fripp et al., 1985), which are similar to the results from this present study. Moreover, regular exercise can also improve physiological health such as decreasing blood pressure (Alpert and Wilmore, 1994), increasing physical fitness (Gutin et al., 1996; Ignico and Mahon, 1995), and decreasing the degree of obesity in children (Brownell and Kaye, 1982; Sasaki et al., 1987). Physical activity among adolescents is consistently related to higher levels of self-esteem and lower levels of stress (Calfas and Taylor, 1994) Researchers have reported that various factors positively associated with physical activity among young people include confidence in one's ability to engage in exercise (Reynolds et al., 1990; Zakarian et al., 1994), perceptions of physical or sport competence (Ferguson et al., 1989; Tappe et al., 1990), having positive attitudes toward physical education and enjoying physical activity (Stucky-Ropp and DiLorenzo, 1993; Tinsley et al., 1995).

The results of this study suggest that the 8-week PEC was a key factor in improving and rehabilitating the psychosocial health of multicultural children. In addition, research supports the importance of social networks in maintaining health and reducing acculturation stress. Recreational activities have the potential to increase social involvement and develop friendships (Kim, 2015). Social, cultural, and physical environmental factors can act as barriers for physical activity (Bodde and Seo, 2009; Kim, 2015; Stanish et al., 2006). Each of these factors is modifiable with the help of agencies and service providers. In particular, the physical environment and behavioral attributes can be modified for children from multicultural families. Thus, physical education should be provided to multicultural children as an effective way to deal with psychosocial problems and improve physical health.

\section{CONFLICT OF INTEREST}

No potential conflict of interest relevant to this article was reported. 


\section{ACKNOWLEDGMENTS}

This research was supported by the 2016 Hanseo University Research Grant.

\section{REFERENCES}

Adlard PA, Cotman CW. Voluntary exercise protects against stress-induced decreases in brain-derived neurotrophic factor protein expression. Neuroscience 2004;124:985-992.

Alpert BS, Wilmore JH. Physical activity and blood pressure in adolescents. Pediatr Exerc Sci 1994;6:361-380.

American College of Sports Medicine. ACSM's Guidelines for exercise testing and prescription. 6th ed. Philadelphia: Lippincott Williams \& Wilkins; 2000.

Armstrong N, Simons-Morton B. Physical activity and blood lipids in adolescents. Pediatr Exerc Sci 1994;6:381-405.

Bailey AW, Fernando IK. Routine and project-based leisure, happiness, and meaning in life. J Leis Res 2012;44:139-154.

Banks JA. An introduction to multicultural education, 5th ed. Cambridge: Pearson; 2013.

Bar-Or O, Baranowski T. Physical activity, adiposity, and obesity among adolescents. Pediatr Exerc Sci 1994;6:348-360.

Becchetti L, Ricca EG, Pelloni A. The relationship between social leisure and life satisfaction: causality and policy implications. Soc Indic Res 2012;108:453-490.

Berkowitz RI, Agras WS, Korner AF, Kraemer HC, Zeanah CH. Physical activity and adiposity: a longitudinal study from birth to childhood. J Pediatr 1985;106:734-738

Berry JW. A psychology of immigration. J Soc Issue 2001;57:615-631.

Berry JW. Acculturation: living successfully in two cultures. Int J Intercult Relat 2005;29:697-712

Bodde AE, Seo DC. A review of social and environmental barriers to physical activity for adults with intellectual disabilities. Disabil Health J 2009;2:57-66.

Brownell KD, Kaye FS. A school-based behavior modification, nutrition education, and physical activity program for obese children. Am J Clin Nutr 1982;35:277-283.

Calfas KJ, Taylor WC. Effects of physical activity on psychological variables in adolescents. Pediatr Exerc Sci 1994;6:406-423.

Cha JY, Kim JH, Hong J, Choi YT, Kim MH, Cho JH, Ko IG, Jee YS. A 12week rehabilitation program improves body composition, pain sensation, and internal/external torques of baseball pitchers with shoulder impingement symptom. J Exerc Rehabil 2014;10:35-44.

Dixon JC, Rosenbaum MS. Nice to know you? Testing contact, cultural, and group threat theories of anti-black and anti-Hispanic stereotypes. Soc Sci Q 2004;85:257-280.

Donnelly JE, Blair SN, Jakicic JM, Manore MM, Rankin JW, Smith BK; American College of Sports Medicine. American College of Sports Medicine Position Stand. Appropriate physical activity intervention strategies for weight loss and prevention of weight regain for adults. Med Sci Sports Exerc 2009;41:459-471.

Dotson CO, Ross JG. Relationships between activity patterns and fitness. J Phys Educ Recreat Dance 1985;56:86-91.

Dunn AL, Trivedi MH, O’Neal HA. Physical activity dose-response effects on outcomes of depression and anxiety. Med Sci Sports Exerc 2001;33(6 Suppl):S587-597.

Ferguson KJ, Yesalis CE, Pomrehn PR, Kirkpatrick MB. Attitudes, knowledge, and beliefs as predictors of exercise intent and behavior in schoolchildren. J Sch Health 1989;59:112-115.

Fripp RR, Hodgson JL, Kwiterovich PO, Werner JC, Schuler HG, Whitman V. Aerobic capacity, obesity, and atherosclerotic risk factors in male adolescents. Pediatrics 1985;75:813-818.

Gutin B, Cucuzzo N, Islam S, Smith C, Stachura ME. Physical training, lifestyle education, and coronary risk factors in obese girls. Med Sci Sports Exerc 1996;28:19-23.

Ignico AA, Mahon AD. The effects of a physical fitness program on lowfit children. Res Q Exerc Sport 1995;66:85-90.

Janssen I, Heymsfield SB, Ross R. Application of simple anthropometry in the assessment of health risk: implications for the Canadian Physical Activity, Fitness and Lifestyle Appraisal. Can J Appl Physiol 2002; 27:396-414.

Kang WS. The influence of participation in intramural sports of elementary school's student on the reduction of stress [master's thesis]. Gongju: Gongju National University; 2004.

Kim CW. Narrative inquiry on experience of culturally diverse students in physical education. Korean J Soc Sport 2011;24:1-21.

Kim KS. A study on the realities of child education in a multicultural family in Korea [master's thesis]. Seoul: Seoul National University; 2006.

Kim MH, Choi YT, Jee YS, Eun D, Ko IG, Kim SE, Yi ES, Yoo J. Reducing the frequency of wearing high-heeled shoes and increasing ankle strength can prevent ankle injury in women. Int J Clin Pract 2015; 69:909-910

Kim YS. Minimizing acculturative stress in leisure for children from multicultural family: application of Peterson and Gunn's leisure education model. J Sport Leis Stud 2015;59:11-19.

Kwon MH. Direction of movement in multicultural society. Sport Sci 2008:103:2-8

Lee EA. Development and validation of elementary school aggression scale [master's thesis]. Busan: Kyungsung University; 2011. 
Lee J, Koeske GF, Sales E. Social support buffering of acculturative stress: a study of mental health symptoms among Korean international students. Int J Intercult Relat 2004;28:399-414.

Léger L, Gadoury C. Validity of the $20 \mathrm{~m}$ shuttle run test with $1 \mathrm{~min}$ stages to predict $\mathrm{VO}_{2}$ max in adults. Can J Sport Sci 1989;14:21-26.

Léger LA, Lambert J. A maximal multistage 20-m shuttle run test to predict $\mathrm{VO}_{2}$ max. Eur J Appl Physiol Occup Physiol 1982;49:1-12.

McLaren LM. Anti-immigrant prejudice in Europe: Contact, threat theory, and preferences for the exclusion of immigrants. Social Force 2003;81:909-936.

Min HK. Effect of emotional intelligence on social development and academic achievement of elementary school students [master's thesis]. Chuncheon: Chuncheon National University; 2005.

Mori S. Addressing the mental health concerns of international students. J Couns Dev 2000;78:137-144.

Newman DB, Tay L, Diener E. Leisure and subjective well-being: a model of psychological mechanisms as mediating factors. J Happiness Stud 2013;15:555-578

Park HS. A grounded theory approach on the school dropout of adolescents in Korea. Korean J Soc Welf 2003;53:75-104.

Park NS. Multicultural education through social studies: citizenship education on multicultural society. Soc Stud Educ 2000;33:107-177.

Pate RR, Ross JG. Factors associated with health-related fitness. J Physical Educ Recreat Dance 1987;58:93-97.

Pil-Byung C, Shin-Hwan Y, Il-Gyu K, Gwang-Suk H, Jae-Hyun Y, HanJoon L, Sung-Eun K, Yong-Seok J. Effects of exercise program on appetite-regulating hormones, inflammatory mediators, lipid profiles, and body composition in healthy men. J Sports Med Phys Fitness 2011;51:654-663.

Reynolds KD, Killen JD, Bryson SW, Maron DJ, Taylor CB, Maccoby N, Farquhar JW. Psychosocial predictors of physical activity in adolescents. Prev Med 1990;19:541-551.

Rudmin F. Constructs, measurements and models of acculturation and acculturative stress. Int J Intercult Relat 2009;33:106-123.

Russo-Neustadt A, Beard RC, Cotman CW. Exercise, antidepressant medications, and enhanced brain derived neurotrophic factor expression. Neuropsychopharmacology 1999;21:679-682.

Sallis JF, McKenzie TL, Alcaraz JE. Habitual physical activity and health-related physical fitness in fourth-grade children. Am J Dis Child 1993;147:890-896.
Sallis JF, Patrick K. Physical activity guidelines for adolescents: consensus statement. Pediatr Exerc Sci 1994;6:302-314.

Sasaki J, Shindo M, Tanaka H, Ando M, Arakawa K. A long-term aerobic exercise program decreases the obesity index and increases the high density lipoprotein cholesterol concentration in obese children. Int J Obes 1987;11:339-345.

Spear BA, Barlow SE, Ervin C, Ludwig DS, Saelens BE, Schetzina KE, Taveras EM. Recommendations for treatment of child and adolescent overweight and obesity. Pediatrics 2007;120 Suppl 4:S254-288.

Stanish HI, Temple VA, Frey GC. Health-promoting physical activity of adults with mental retardation. Ment Retard Dev Disabil Res Rev 2006;12:13-21.

Steffen PR, Smith TB, Larson M, Butler L. Acculturation to Western society as a risk factor for high blood pressure: a meta-analytic review. Psychosom Med 2006;68:386-397.

Stucky-Ropp RC, DiLorenzo TM. Determinants of exercise in children. Prev Med 1993;22:880-889.

Tabata I, Nishimura K, Kouzaki M, Hirai Y, Ogita F, Miyachi M, Yamamoto K. Effects of moderate-intensity endurance and high-intensity intermittent training on anaerobic capacity and VO2max. Med Sci Sports Exerc 1996;28:1327-1330.

Tadmor CT, Tetlock PE. Biculturalism: a model of the effects of second-culture exposure on acculturation and integrative complexity. J Cross Cult Psychol 2006;37:173-190.

Tappe MK, Duda JL, Menges-Ehrnwald P. Personal investment predictors of adolescent motivational orientation toward exercise. Can J Sport Sci 1990;15:185-192.

Temple VA, Stanish HI. Physical activity and persons with intellectual disability: some considerations for Latin America. Salud Publica Mex 2008;50 Suppl 2:s185-193.

Tinsley BJ, Holtgrave DR, Reise SP, Erdley C, Cupp RG. Developmental status, gender, age, and self-reported decision-making influences on students' risky and preventive health behaviors. Health Educ Q 1995; 22:244-259.

Wang M, Wong MC. Leisure and Happiness: Evidence from International Survey Data. J Happiness Stud 2014;15:85-118.

Zakarian JM, Hovell MF, Hofstetter CR, Sallis JF, Keating KJ. Correlates of vigorous exercise in a predominantly low SES and minority high school population. Prev Med 1994;23:314-321. 